\title{
Agrobacterium-mediated Genetic Transformation of Mungbean (Vigna radiata (L.) Wilczek)
}

\section{Mohammad Nurul Islam* and Kazi Tariqul Islam}

Plant Breeding and Biotechnology Laboratory, Department of Botany, University of Dhaka, Dhaka-1000, Bangladesh

Kew words: Mungbean, Vigna radiata, BINA mug-5,Transformation, nptII, GUS

Mungbean (Vigna radiata (L.) Wilczek) is one of the most important pulse crops in world agriculture for its protein rich edible seeds. It is widely cultivated in Bangladesh and regarded as a quality pulse. Moreover, it contributes to improve the soil fertility by fixing atmospheric nitrogen and accumulating significant amount of organic matter to the soil. The demand for this crop has been steadily increasing in the Indian subcontinent and it has been playing an important role in fulfilling the nutritional requirements of population of Bangladesh. But the mungbean production is failing to support its optimum requirements because of various biotic and abiotic stresses. In some years losses exceed more than 50\% due to incidence of different pests and diseases such as Yellow Mosaic Virus, Cercospora leaf spot etc. (Poehlman 1991 and Bose 1991).

Therefore, improvement of this crop is essential to obtain desired agronomic performance such as disease resistance, high productivity, enhanced nutritional value and other essential agronomic qualities of this crop. Limiting factors for the improvement of this crop through conventional breeding techniques are the existence of narrow genetic base and absence of resistant gene/s of interest in the existing germplasms. Genetic transformation can supplement traditional breeding programs to introduce desired gene/s into this crop. Therefore, it is necessary to establish a suitable transformation protocol for local mungbean varieties to develop disease and pest resistant lines of mungbean which are not available in existing mungbean germplasms.

In the past few years, some progress has been made regarding the in vitro regeneration of mungbean (Jaiwal et al. 2001, Sarker and Siddiqua 2004, Mahalakshmi et al. 2006), but very little success has been reported regarding genetic transformation of mungbean through Agrobacterium-mediated genetic transformation of local varieties of mungbean (Hoque et al. 2007). Considering the importance of mungbean as a major protein yielding legume crop in Bangladesh,

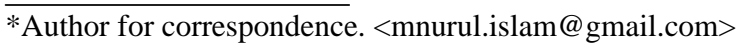


the present investigation attempts were made to develop reproducible Agrobacterium-mediated genetic transformation protocol for local varieties of mungbean. During the present investigation previously reported regeneration protocols established by Hoque et al. (2007) have been used.
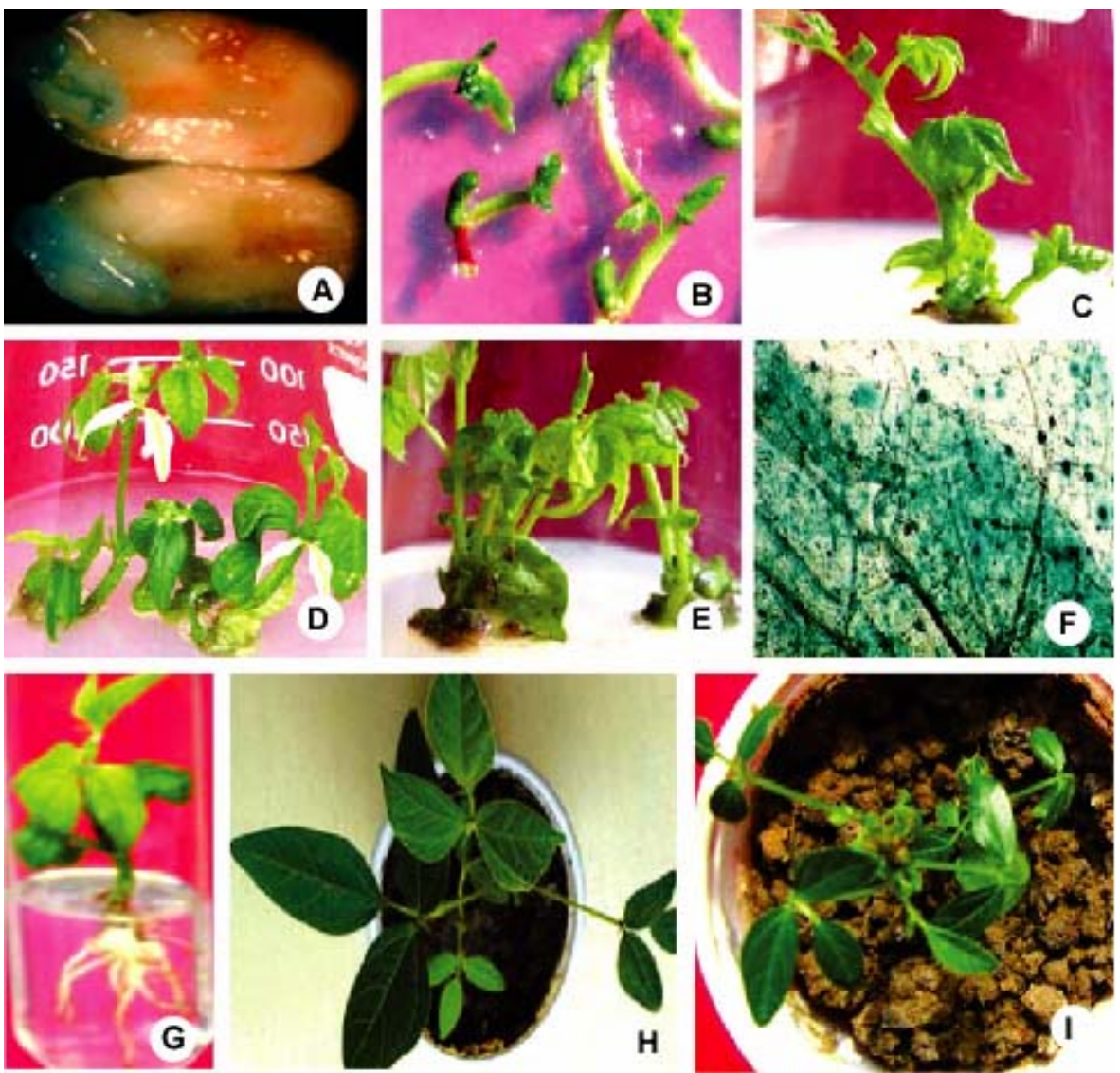

Fig. 1. Genetic transformation and development putative transgenic plants of mungbean. A. Histochemical localization of GUS activity at the cut surface of CAEA explants. B. Shoot initiation on MS medium with $1.0 \mathrm{mg} / \mathrm{l}$ BAP from CAEA explants. C. Multiple shoots initiation and elongation on the same media. D. Non-transformed shoots showing albinism on the kanamycin-containing selection medium. E. Putative transformed shoots growing on the kanamycin-containing selection medium. F. Chimeric expression of GUS gene as observed in leaf. G, H and I. Rooting of putative transformed shoots and establishment of a To plantlet into soil.

Seeds of BINA mug- 5 variety were germinated on water-agar media $(0.8 \%$ agar) without any sucrose to get explants for tissue culture and transformation experiments. Two different types of explants, namely cotyledonary leaf and cotyledon attached with embryonal axis (CAEA) were used in different experiments. Cotyledonary leaf explants were collected from 1 - 2 days old aseptically grown germinated seeds and CAEA explants were collected from the surface sterilized germinated seeds grown overnight. 
CAEA and cotyledonary leaf explants were infected with A. tumefaciens strain LBA4404 harboring the binary plasmid pBI121 containing gusA and neomycinphosphotransferase (nptII) marker genes to determine their transformation ability. Between two explants, CAEA showed better response towards transformation than the cotyledonary leaf. Maximum transformation of CAEA explants was obtained following $45 \mathrm{~min}$ of infection with Agrobacterial suspension having an OD of 1.3 at $600 \mathrm{~nm}$ and $72 \mathrm{~h}$ of cocultivation as judged by transient GUS assays. Following cocultivation, explants were subjected to transient GUS histochemical assay according to Jefferson et al. (1987) to determine the infection efficiency. Conspicuous blue color zones were detected at the regeneration point of the cocultured CAEA (Fig. 1A) and cotyledonary leaf explants as well as the internal tissues were observed under the stereomicroscope. After cocultivation, explants were washed with sterile distilled water and subcultured in the best shoot regeneration medium reported earlier (Hoque et al. 2007). In the present investigation, antibiotic selection pressure was delayed until shoot induction. Putatively transformed shoots were selected by gradually increasing kanamycin concentration (Figs. 1B,C). When the regenerated shoots attained a height of about $1.0-1.5 \mathrm{~cm}$, they were transferred on to MS containing lower concentrations of kanamycin $(50 \mathrm{mg} / \mathrm{l})$ as a selectable agent. The concentration was gradually increased up to $200 \mathrm{mg} / \mathrm{l}$ with each subculture at 12 - 15 days interval. During each subculture, the albino or deep brown dead tissue bearing shoots were discarded and only green shoots and shoot buds were subcultured in fresh medium with higher concentrations of selectable (kanamycin) agent (Figs. 1D,E). Sarker et al. (2003) develpoed putative transgenic shoots from BM-2 and BM-4 varieties of lentil as described above. Experiments were carried out to observe the stable expression of GUS gene in the shoots developed under selection pressure. Different parts of developing shoots such as shoot tips and petiole-attached leaves were subjected to GUS histochemical assay to detect the expression of integrated GUS gene. The presence of patches of conspicuous blue color zones on different parts of the shoot i.e, leaf, stem, petiole etc. (Fig. 1F), confirmed the integration and expression of transgene into the genome of BINA mung5. Shoots that survived during selection process and developed GUS positive blue colored zones in different parts were induced to rooting in the medium containing $0.5 \mathrm{mg} / \mathrm{l}$ IBA maintaining selection pressure with $200 \mathrm{mg} / \mathrm{l}$ kanamycin. None of these shoots produce roots. In chickpea putative transformed shoots were successfully rooted without any selection pressure (Fontana et al. 1993 and Kar et al. 1996). Therefore, rooting of transformed shoots was carried out without selection pressure during the present study. Healthy rooted transformed plants (To) (Fig. 1G) were subjected to hardening to establish the plants in normal environ-mental condition primarily in plastic pots and finally in earthen pots (Figs. 1H,I). 
It can be concluded that the protocol of Agrobacterium-mediated genetic transformation developed during the present investigation using screenable marker genes like gus A and selectable marker genes like nptII is applicable for the transformation of locally grown mungbean varieties. Using this transformation protocol, future programs can be undertaken to transfer useful candidate genes conferring to disease, insect and pest resistance in local mungbean cultivars.

\section{Acknowledgements}

Authors are extremely grateful to Professor A.S. Islam and Professor M.M. Haque for critically going through the manuscript and suggesting necessary corrections. The authors are also thankful to BINA authorities for providing seed materials for the present investigation.

\section{References}

Bose M (1991) In vitro plant regeneration from different explants of mungbean (Vigna radiata (L.) Wilczek). M.Sc. Thesis, Department of Botany, University of Dhaka.

Fontana GS, Santini L, Caretto S, Frugis G and Mariotti D (1993) Genetic transformation in the grain legume Cicer arietinum L. (Chickpea). Plant Cell Reports 21: 194-198.

Hoque MI, Zahan MM and Sarker RH (2007) In vitro plant Regeneration of Mungbean (Vigna radiate (L.) Wilckzek). Plant Tissue Cult. \& Biotech. 17(2): 209-216.

Jaiwal PK, Kumari R, Ignacimuthu S, Potrykus I and Sautter C (2001) Agroabcterium tumefaciens-mediated genetic transformation of mungbean (Vigna radiata (L.) Wilczek) recalcitrant grain legume. Plant Sci. 161: 239-247.

Jefferson RA, Kavanagh TA and Bevan MW (1987) GUS fusion: $\beta$-glucuronidase as a sensitive and versatile gene fusion marker in higher plants. EMBO J. 6: 3901-3907.

Kar S, Johanson TM, Nayak P and Sen SK (1996) Efficient transgenic plant regeneration through Agrobacterium-mediated transformation of chickpea (Cicer arietinum L.). Plant Cell Reports 16: 32-37.

Mahalakshmi LS, Leela T, Kumar SM, Kumar BK, Naresh B and Devi P (2006) Enhanced genetic transformation efficiency of mungbean by use of primary leaf explants. Curr. Sci. 91(1): 93-99.

Poehlman JM (1991) The mungbean. Oxford and IBH Publishing Co. Pvt. Ltd., New Delhi, India.

Sarker RH, Mustafa BM, Biswas A, Mahbub S, Nahar M, Hashem R and Hoque MI (2003) Agrobacterium-mediated transformation of lentil (Lens culinaris Medik.). Plant Tissue Cult. 13(1): 1-12.

Sarker RH and Siddiqua K Murshida (2004) In vitro plant regeneration and preliminary stides on Agrobacterium-mediated genetic transformation of mungbean. In: In vitro application in crop improvement. Mujib, A., Cho, M., Predieri, S. and Banerjee, S. (eds). Science Publishers, Inc. 155-169. 